\title{
Radio studies of relativistic SN 2009bb
}

\author{
Alak Ray ${ }^{1}$, Naveen Yadav ${ }^{1}$, Sayan Chakraborti ${ }^{2}$, Alicia Soderberg ${ }^{2}$ \\ and Poonam Chandra ${ }^{3}$ \\ ${ }^{1}$ Tata Institute of Fundamental Research, Mumbai 400005, India \\ email: akr@tifr.res.in, nyadav@tifr.res.in \\ ${ }^{2}$ Harvard-Smithsonian Center for Astrophysics, Cambridge, MA 02138, USA \\ ${ }^{3}$ National Centre for Radio Astrophysics, TIFR, Pune 411007, India
}

\begin{abstract}
A local sub-population of type Ib/c supernovae (stripped envelope SNe) with mildly relativistic outflows have been detected as sub-energetic Gamma Ray Bursts (GRBs) or X-ray Flashes (XRFs) and as radio afterglows without detected GRB counterpart. SN 2009bb belongs to the last class of objects. The long term radio observations with $(\mathrm{J}) \mathrm{VLA}$ and GMRT of this SN map the dynamics of the relativistic ejecta characteristic of Central Engines associated with GRBs. We present here GMRT observations of this SN from October 2009 onwards.
\end{abstract}

Keywords. shock waves, supernovae, radio continuum: general, circumstellar matter

Relativistic bulk motion of matter is implied (Piran 1999, Piran 2004) in long duration gamma ray bursts (GRBs) that are linked to core-collapse explosion of a stripped envelope massive star (Paczynski 1986, Woosley 1993). In the collapsar model (MacFadyen \& Woosley 1999), matter flows towards a newly formed black hole or rapidly spinning, highly magnetized neutron star, constituting the central engine from which powerful jets are launched along the spin axis which plow through the collapsing star eventually attaining relativistic speeds and producing the GRB. The association of energetic core collapse SN 1998bw with the underluminous low redshift $(z=0.0085)$ GRB 980425, as well as spectroscopic identification of SN features well after the GRB event, established the SNGRB connection (Hjorth \& Bloom 2012 and references therein). These SNe belong to a rare subclass of type Ibc SNe called the broad-line Ic's. The central engine driven objects have been discovered mainly through their concomitant gamma-ray emission, but the discovery of luminous radio emission from the type Ibc SN 2009bb that was undetected in gamma-rays despite extensive search led to the measurement of a substantial relativistic outflow that must be powered by the central engine (Soderberg et al. 2010). The outflow speed was measured by combining observations of SN 2009bb from the VLA and the GMRT at multiple epochs. These are well fitted by Synchrotron Self Absorption (SSA) models which can lead to radiosphere locations interpreted from a combination of spectral peak frequencies and fluxes.

We report GMRT observations of SN 2009bb from October 2009. The GMRT and earlier VLA data are plotted in Fig 1.

The radius evolution with time measured from the SSA spectrum showed the SN had mildly relativistic ejecta (Soderberg et al. 2010, Chakraborti \& Ray 2011). The magnetic field amplified by the shock and how it evolves with time and the role of such central engine driven explosions as accelerators of Ultra High Energy Cosmic Ray accelerators are described in Chakraborti et al. 2011. 
Table 1. GMRT observations of SN 2009bb

\begin{tabular}{cccc}
\hline Observation Dates & $\begin{array}{c}\text { Days after } \\
\text { explosion }\end{array}$ & $\begin{array}{c}\text { Frequency } \\
(\mathrm{MHz})\end{array}$ & $\begin{array}{c}\text { Flux density } \\
\text { (mJy) }\end{array}$ \\
\hline 22Oct-01Nov 2009 & $218-223$ & 325 & $12.0 \pm 0.26$ \\
& & 610 & $6.3 \pm 0.70$ \\
& & 1280 & $4.4 \pm 0.30$ \\
09Feb-18Feb 2010 & $328-337$ & 325 & $7.2 \pm 0.29$ \\
& & 610 & $3.6 \pm 0.05$ \\
& & 1280 & $1.2 \pm 0.07$ \\
17Apr-25Apr 2010 & $395-403$ & 325 & $7.4 \pm 0.34$ \\
& & 610 & $3.6 \pm 0.09$ \\
& & 1280 & $1.9 \pm 0.05$ \\
26Dec-31Dec 2010 & $648-653$ & 325 & $6.1 \pm 0.12$ \\
& & 610 & $1.9 \pm 0.05$ \\
& & 1280 & $0.9 \pm 0.05$ \\
26Apr-05May 2011 & $769-778$ & 325 & $4.6 \pm 0.19$ \\
& & 610 & $1.4 \pm 0.08$ \\
& & 1280 & $0.7 \pm 0.05$ \\
04Jan-13Jan 2012 & $1022-1031$ & 325 & $5.9 \pm 0.18$ \\
& & 610 & $1.4 \pm 0.06$ \\
& & 1280 & $0.7 \pm 0.03$ \\
17Jul-20Jul 2012 & \multirow{2}{*}{$1217-1220$} & 325 & $4.4 \pm 0.15$ \\
& & 610 & $2.0 \pm 0.04$ \\
\hline
\end{tabular}

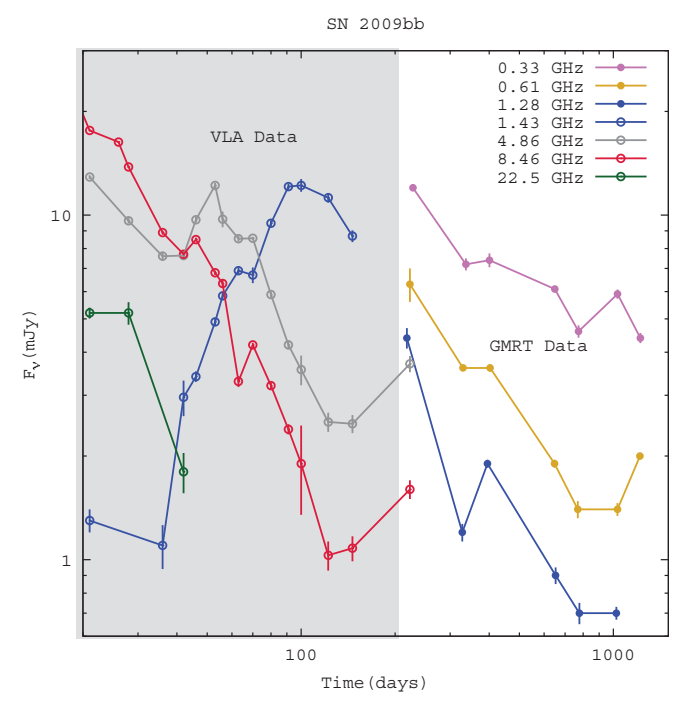

Figure 1. Radio light curves of SN 2009bb at indicated frequencies

\section{References}

Chakraborti, S. \& Ray, A. 2011, ApJ, 729, 57

Chakraborti, S., Ray, A., Soderberg, A. M., \& Loeb, A. 2011, Nature Communications, 2, 175

Hjorth, J. \& Bloom, J. S. 2012, in "Gamma-Ray Bursts", Cambridge Astrophysics Series 51, eds. C. Kouveliotou, et al., Cambridge University Press (Cambridge), p. 169

MacFadyen, A. I. \& Woosley, S. E. 1999, Ap J, 424, 262

Paczynski, B. 1986, Ap J, 308, L43

Piran, T. 1999, Phys. Reports, 314, 575

Piran, T. 2004, Rev. Mod. Phys, 76, 1143

Soderberg, A. M., Chakraborti, S., et al. 2010, Nature, 463, 513

Woosley, S. E. 1993, ApJ, 405, 273 